DOI 10.35381/cm.v7i3.593

\title{
El desempeño directivo en las instituciones educativas desde la innovación tecnológica de control y monitoreo
}

\section{The managerial performance in educational institutions from the technological innovation of control and monitoring}

Carmen Rosa Sánchez-Tejada

P7000088912@ucvvirtual.edu.pe

Universidad Cesar Vallejo, Trujillo

Perú

https://orcid.org/0000-0002-0525-2261

Zila Isabel Esteves-Fajardo

zila.estevesf@ug.edu.ec

Universidad de Guayaquil, Guayaquil

Ecuador

https://orcid.org/0000-0002-2283-5370

Gladys Lola Luján-Johnson

ljohnsongl@ucvvirtual.edu.pe

Universidad Cesar Vallejo, Trujillo

Perú

https://orcid.org/ 0000-0002-4727-6931

Mónica Mendoza-Gómez

monica.mendoza@educacion.gob.ec

Universidad de Guayaquil, Guayaquil

Ecuador

https://orcid.org/0000-0003-1051-4972

Recibido: 15 de agosto de 2021

Aprobado: 15 de noviembre de 2021 
Carmen Rosa Sánchez-Tejada; Zila Isabel Esteves-Fajardo; Gladys Lola Luján-Johnson; Mónica Mendoza-Gómez

\title{
RESUMEN
}

En la presente investigación se formuló como objetivo general diagnosticar el desempeño directivo en las instituciones educativas desde la innovación tecnológica de control y monitoreo. Apoyado en la metodología cuantitativa la cual produce datos descriptivos, que se originan por la recolección de datos. Además, se aplica el método inductivodeductivo, el cual sugiere que para encontrar una verdad se deben buscar los hechos y no basarse en meras especulaciones. Desde un diseño bibliográfico de tipo documental, cuya pesquisa desde la deliberación crítica, se construyó el método de análisis del fenómeno de estudio. La investigación utilizó la encuesta como técnica y el cuestionario como instrumento para recopilar información. Se recomienda a los directivos de las instituciones educativas, trabajar en acuerdo con el personal docente, la implementación de herramientas de evaluación que permitan identificar fortalezas y debilidades de la labor pedagógica con el objetivo de optimizarla, proponiendo la ejecución de proyectos de innovación.

Descriptores: Innovación educacional; gestión de la educación; control de gestión. (Tesauro UNESCO).

\begin{abstract}
In this research, the general objective was formulated to diagnose the managerial performance in educational institutions from the technological innovation of control and monitoring. Supported by the quantitative methodology which produces descriptive data, which originate from data collection. In addition, the inductive-deductive method is applied, which suggests that to find a truth, the facts must be sought and not based on mere speculation. From a bibliographic design of a documentary type, whose research from critical deliberation, the method of analysis of the study phenomenon was built. The research used the survey as a technique and the questionnaire as an instrument to collect information. The directors of educational institutions are recommended to work in agreement with the teaching staff, the implementation of evaluation tools that allow identifying strengths and weaknesses of the pedagogical work with the aim of optimizing it, proposing the execution of innovation project.
\end{abstract}

Descriptors: Educational innovation; management of education; management control. (UNESCO Thesaurus). 
Carmen Rosa Sánchez-Tejada; Zila Isabel Esteves-Fajardo; Gladys Lola Luján-Johnson;

Mónica Mendoza-Gómez

\section{INTRODUCCIÓN}

La innovación de la gestión educativa, se presenta como uno de los grandes desafíos de la trasformación de la educación, no obstante, las naciones en Latinoamérica afrontan inconvenientes que afectan permanentemente sus sistemas educativos (Borden, 2015, p. 1). Entre los principales problemas de los sistemas educativos se tiene: La necesidad de efectuar estrategias para optimizar la gestión escolar, además, es preciso que se promueva la comprensión de los nuevos conceptos sobre "autonomía" y "participación" del directivo, por último, que las estrategias de capacitación y formación a los gerentes educativos, sean apropiadas a las nuevas competencias en las que se desempeñan (Schettini y Pozner, 2001, p.67). Al respecto, Antúnez (2000) sostiene que el rol directivo resulta un aspecto complejo, pues, su accionar tiene origen en demandas de los variados componentes de la escuela y el contexto a la que pertenece esta, como propósitos, recursos humanos, materiales, económicos, valores y creencias. (p. 30). En este contexto, Puiggros (2020) afirma que la situación se presenta más compleja al evidenciar que, los sistemas educativos en América Latina acogieron la pandemia disminuidos y atrasados en la implementación de cambios que se requerían con suma urgencia. (p. 33). Sin embargo, los constantes cambios en el contexto educativo mundial, han demandado que los roles que desarrollan los gerentes educativos evolucionen hacia una gestión con mayor independencia y orientada a la optimización de los logros de aprendizaje de los niños y jóvenes (Bolívar, 2010.p. 33). Al respecto, Casassus (2000) sostiene que es necesario promover el aspecto pedagógico en la gestión educativa, lo cual implica reflexionar sobre la gestión educativa para que esta no se circunscriba a la dirección de los recursos, además debe gestionarse desde el aspecto de liderazgo pedagógico. ( $p$. 24). 
Carmen Rosa Sánchez-Tejada; Zila Isabel Esteves-Fajardo; Gladys Lola Luján-Johnson; Mónica Mendoza-Gómez

En esta línea, Ugarte, Arguedas y Ángeles (2012), señalan que, en los últimos años el rol del directivo en las escuelas ha ido visibilizándose producto de la implementación de los procesos de descentralización educativa (p. 112); proceso que requería ser implementado con premura. En el ámbito educativo peruano, Guadalupe, León, Rodríguez y Vargas (2017) sostienen que:

El ministerio de educación toma decisiones de lo que debe suceder en las escuelas, de ahí que los directores no puedan ser líderes pedagógicos, pues, destinan la mayor parte de su tiempo a responder los pedidos del propio MINEDU, por tanto, no se considera la posibilidad de convertir a la escuela en un espacio fundamental de la gestión. (p. 242).

En esta línea, Cuglievan y Rojas (2007) señalan que:

Si bien los roles y funciones de quienes dirigen las escuelas en nuestro país están transformándose desde disposiciones legales, estos no se implementan al interior de las instituciones, generando la ruptura entre la gestión y las prácticas institucionales, limitando el logro de los propósitos y fines previstos. (p. 337).

En este sentido, según la resolución ministerial (RM N 620 -2019 -MINEDU), sobre el "Plan de gobierno digital del Minedu en los años 2019 -2022", prioriza la necesidad de determinar, examinar y optimar los procedimientos de la institución, con el propósito de afirmar su alineamiento con la estrategia institucional y alcanzar las metas en favor de la nación. Además, en el Proyecto Educativo Nacional (PEN), al 2036, se señala que para para la mejora de la educación, es necesaria la Innovación mediante la tecnología para facilitar los procesos educativos. También, sobre la labor directiva, se recoge la necesidad y la preocupación de la población que sueña tener un sistema educativo donde los equipos directivos realicen un trabajo articulado tanto de la gestión pedagógica y administrativa. 
Carmen Rosa Sánchez-Tejada; Zila Isabel Esteves-Fajardo; Gladys Lola Luján-Johnson; Mónica Mendoza-Gómez

La unidad de gestión educativa local (UGEL) Piura, cuenta con más de 146,000 estudiantes, 8 mil docentes y 1,500 instituciones educativas, y teniendo en cuenta que es una unidad operativa que no administra recursos financieros, resulta muy complicado el control y seguimiento al personal y los procesos educativos en las escuelas de su jurisdicción. Ante esta realidad, se precisa para una mejor gestión de la UGEL Piura, disponer de un sistema que permita controlar y hacer seguimiento a cinco procesos críticos de la Educación como son: 1. Monitoreo y acompañamiento, 2. Asistencia del personal docente y administrativo, 3. Manejo de recursos y materiales, 4. Asistencia de los estudiantes, y 5 . Documentos de gestión y responsabilidades con la UGEL.

Luego de planteado el tema a desarrollar, se formula como objetivo general diagnosticar el desempeño directivo en las instituciones educativas desde la innovación tecnológica de control y monitoreo.

\section{METODOLOGÍA}

La presente investigación utilizó la metodología cuantitativa la cual produce datos descriptivos, que se originan por la recolección de datos. Además, se aplica el método inductivo-deductivo, el cual sugiere que para encontrar una verdad se deben buscar los hechos y no basarse en meras especulaciones, además de partir de afirmaciones generales para llegar a específicas (Dávila, 2006). Desde un diseño bibliográfico de tipo documental, cuya pesquisa desde la deliberación crítica, se construyó el método de análisis del fenómeno de estudio. La investigación utilizó la encuesta como técnica y el cuestionario como instrumento para recopilar información. 


\section{RESULTADOS}

1. Respecto al estado de la labor directiva de los directores de las Instituciones Educativas de la UGEL Piura, se tiene el siguiente análisis de datos. Así mismo, en cuanto a la gestión de las condiciones para la mejora de los aprendizajes: En Planificación Institucional, participación democrática, condiciones operativas que favorecen el aprendizaje, evaluación de la gestión educativa y rendición de cuentas se muestran la siguiente descripción:

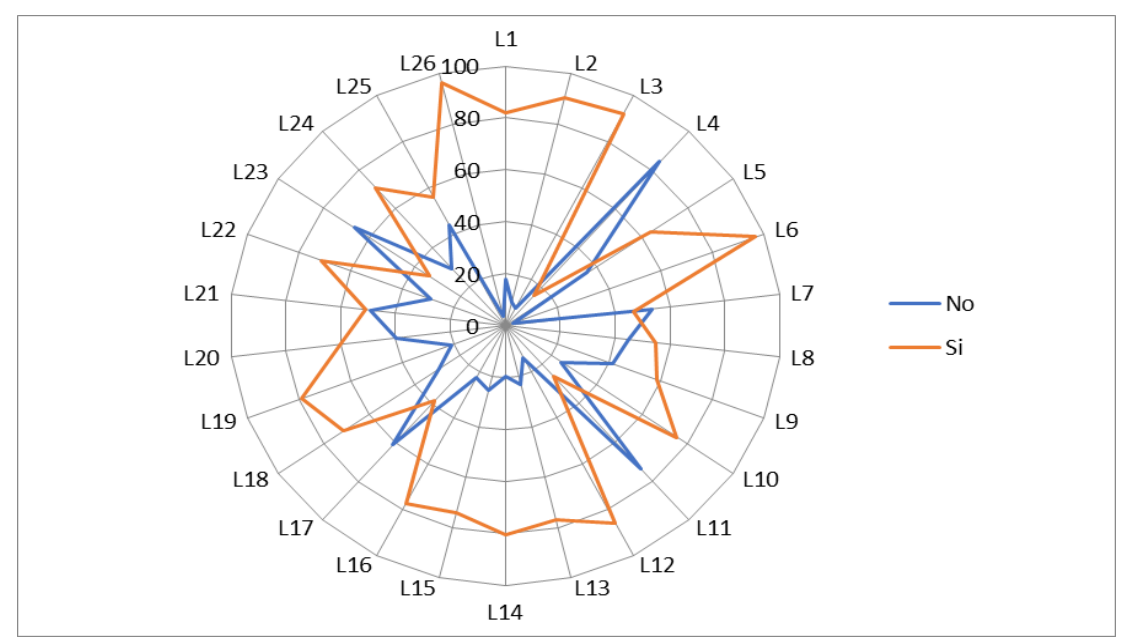

Gráfico 1. Caracterización de la labor directiva con respecto a la gestión de las condiciones para la mejora de los aprendizajes.

Elaboración: Los Autores.

\section{Descripción:}

L1 En el presente año se han desarrollado reuniones para identificar las características de la institución educativa en relación a los procesos pedagógicos, clima escolar e influencia

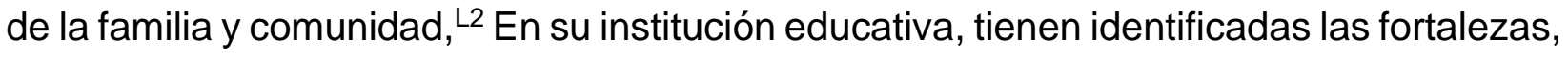
debilidades y necesidades, ${ }^{\mathrm{L} 3} \mathrm{En}$ el presente año se han formulado y/o reajustado los instrumentos de gestión escolar en su institución educativa, ${ }^{4}$ Se ha remitido información a UGEL sobre la elaboración y/o reajuste de los instrumentos de gestión escolar, ${ }^{\mathrm{L} 5}$ En 
Carmen Rosa Sánchez-Tejada; Zila Isabel Esteves-Fajardo; Gladys Lola Luján-Johnson; Mónica Mendoza-Gómez

los instrumentos de gestión se han establecido metas institucionales y de aprendizaje, ${ }^{\mathrm{L} 6}$ La comunidad educativa de su institución está organizada en comisiones, L7 Se ha remitido información a UGEL sobre la conformación de comisiones en la institución educativa, ${ }^{\mathrm{L} 8} \mathrm{El}$ personal docente y administrativo de la institución educativa, es motivado por su participación en el logro de las metas institucionales, ${ }^{\text {L9 }}$ En la institución educativa se propician espacios de integración del personal con la finalidad de promover un clima laboral favorable, L10 Comunica a los docentes, administrativos y estudiantes sus altas expectativas, comprometiéndolos en la búsqueda de mejores desempeños, L11 Utiliza alguna herramienta para la identificación de situaciones conflictivas en la institución educativa, L12 Implementa algún tipo de técnicas de resolución de conflictos a nivel de la institución educativa, ${ }^{\mathrm{L} 13} \mathrm{La}$ institución educativa tiene convenio con alguna institución de la comunidad, L14 En la institución educativa se tienen referenciadas las principales necesidades de infraestructura, equipamiento y materia, ${ }^{\mathrm{L} 15} \mathrm{Se}$ ha cumplido con enviar información de declaración de gastos del programa de "Mi Mantenimiento" a UGEL , L16 Las diversas actividades que se desarrollan en la institución educativa están planificadas de algún documento, L17 Registra el monitoreo a los docentes sobre el cumplimiento de las horas efectivas de clase, ${ }^{\mathrm{L} 18} \mathrm{Hace}$ de conocimiento a la comunidad educativa $\mathrm{y} / \mathrm{o}$ UGEL el uso de los recursos financieros, ${ }^{L 19}$ En la institución educativa se ha conformado la comisión de gestión de recursos financieros, ${ }^{\text {L20 }}$ En la institución educativa se ha conformado la comisión de gestión de riesgos, ${ }^{\mathrm{L} 21}$ Se ha remitido a UGEL el Plan de

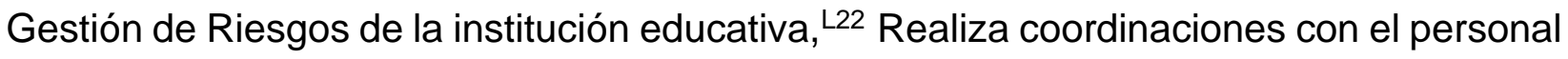
administrativo relacionadas a asuntos institucionales, ${ }^{\mathrm{L} 23}$ Utiliza herramientas pertinentes y oportunas de procesamiento y organización de la información que contribuyen con la toma de decisiones institucionales en favor de la mejora de los aprendizajes, ${ }^{\text {L24 Comunica }}$ la información presentada por los diversos equipos de trabajo institucionales acerca de la marcha de la gestión escolar, ${ }^{\mathrm{L} 25} \mathrm{La}$ institución educativa cuenta con mecanismos de transparencia y rendición de cuentas para hacer públicos los resultados de la escuela y 
los aprendizajes de los estudiantes. ${ }^{2} 26$ En la institución educativa se realiza un seguimiento al avance de los objetivos y/o metas institucionales planificadas.

\section{Análisis}

El gráfico 1 refleja las acciones del proceso de gestión de las condiciones para la mejora de los aprendizajes en un orden de mayor a menor necesidad de atención por las respuestas mayoritaria "NO" por los directores designados y encargados: Primero. El indicador conduce la planificación institucional (4L: 84\%; L5: 36\%). Segundo. El indicador promueve y sostiene la participación democrática de los diversos actores educativos (L11: 73,8\%; L7: 53\%; L8: 45\%). Tercero: El indicador favorece las condiciones operativas que aseguren el aprendizaje de calidad (L17: 61,3\%; L 21: 49,2\%; L20: 39,8\%) y cuarto: el indicador lidera procesos de evaluación de la gestión de la institución educativa y de rendición de cuentas (L23: 66,5\%; L25:44\%; 29,3). 
2. Respecto a la orientación de los procesos pedagógicos para la mejora de los aprendizajes: Promueve y lidera una comunidad de aprendizaje con las y los docentes de su institución educativa y Gestiona la calidad de los procesos pedagógicos al interior de su institución educativa se muestran la siguiente descripción:

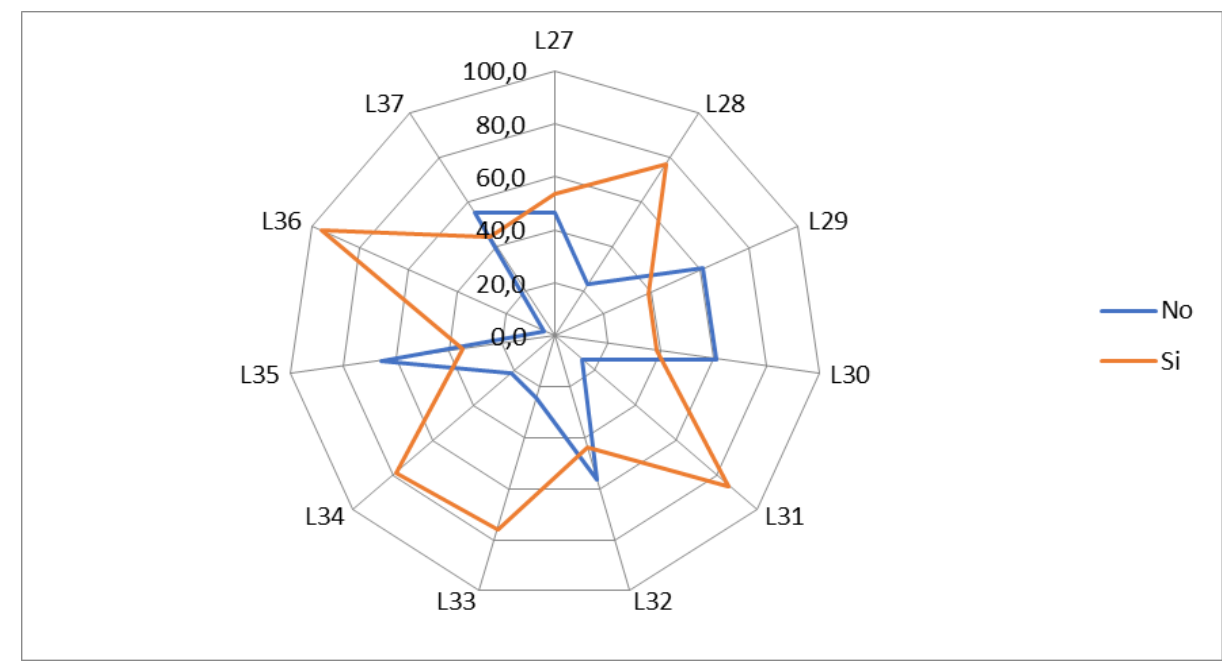

Gráfico 2. Caracterización de la labor directiva con respecto a la orientación de los procesos pedagógicos para la mejora de los aprendizajes.

Elaboración: Los Autores.

\section{Descripción:}

${ }^{\text {L27 }} \mathrm{Ha}$ utilizado herramientas para identificar las fortalezas y debilidades de su personal docente, ${ }^{\mathrm{L} 28} \mathrm{Se}$ ha implementado en la institución educativa mecanismos de trabajo

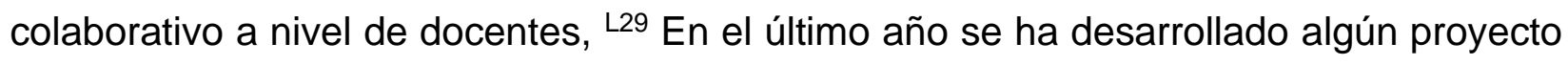
de innovación pedagógica por parte del personal de su institución educativa, ${ }^{\llcorner 30} \mathrm{Ha}$ participado a nivel de UGEL, DREP y/o MINEDU en concursos de proyectos de innovaciones pedagógicas, ${ }^{\llcorner 31}$ Se reúne con los docentes de su institución educativa con la finalidad de elaborar la planificación curricular de experiencias de aprendizaje, L32 Informa a UGEL sobre el trabajo de planificación, implementación y evaluación curricular realizado con los docentes, ${ }^{\mathrm{L} 33} \mathrm{Se}$ han desarrollado en la institución educativa reuniones 
Carmen Rosa Sánchez-Tejada; Zila Isabel Esteves-Fajardo; Gladys Lola Luján-Johnson; Mónica Mendoza-Gómez

de trabajo con los docentes para promover el trabajo colaborativo y por indagación,

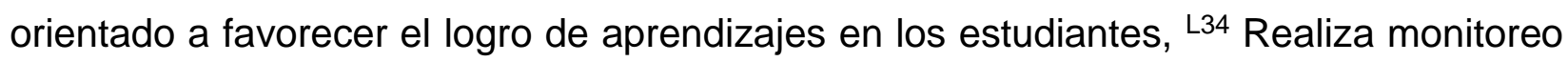
al personal docente sobre el uso de estrategias y recursos metodológicos, uso efectivo del tiempo y los materiales educativos ${ }^{\mathrm{L} 35}$ Convoca a los docentes a reuniones de trabajo para fortalecer los aspectos débiles en su práctica pedagógica, identificados en el monitoreo ${ }^{\mathrm{L} 36}$ Realiza monitoreo al personal docente sobre el uso de metodologías de evaluación, ${ }^{\mathrm{L37} C o n v o c a}$ a los docentes a reuniones de trabajo para fortalecer los procesos de evaluación que necesitan ser mejorados, identificados en el monitoreo

\section{Análisis:}

El gráfico 2 refleja las acciones de la orientación de los procesos pedagógicos para la mejora de los aprendizajes en un orden de mayor a menor necesidad de atención por las respuestas mayoritarias de "NO" por los directores de las instituciones educativas de la UGEL Piura designados y encargados: Primero en el indicador promueve y lidera una comunidad de aprendizaje con las y los docentes de su institución educativa (L27:61\%; L30:61\%; L29:46,6\%); Segundo. En el indicador Gestiona la calidad de los procesos pedagógicos al interior de su institución educativa (L35: 65,4\%; L32: 56,5\%; L37:55,5\%). 
3. Respecto al estado de la innovación tecnológica de control y monitoreo, se tiene el siguiente análisis de datos. Respecto al monitoreo y acompañamiento: Directivos y Docentes monitoreados y acompañados se muestran la siguiente descripción:

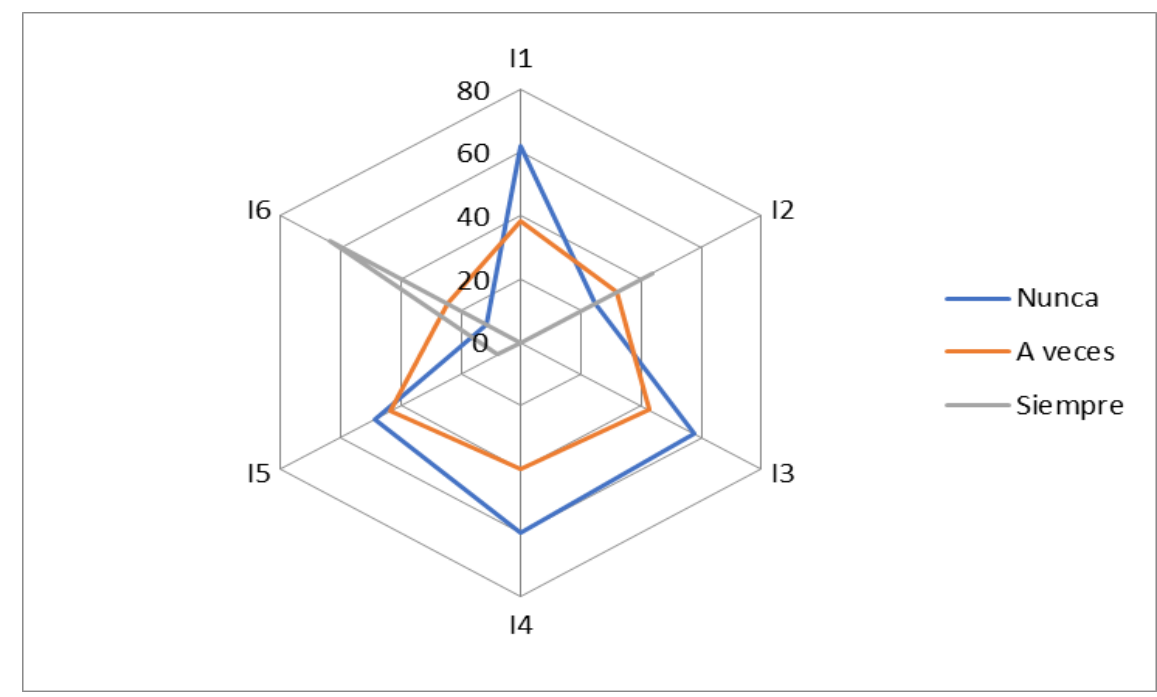

Gráfico 3. Caracterización de la innovación tecnológica de control y monitoreo con respecto al monitoreo y acompañamiento

Elaboración: Los Autores.

\section{Descripción:}

${ }^{11}$ El cronograma de visitas de monitoreo y acompañamiento que realiza el personal de UGEL a los directivos de las IIEE, se visualiza en algún medio virtual, ${ }^{12}$ La UGEL Piura utiliza medios virtuales para registrar el monitoreo y acompañamiento realizada a su instituciones educativa, ${ }^{13}$ Se realiza un seguimiento virtual por parte del personal de UGEL en relación a la primera visita de monitoreo y acompañamiento realizada en su institución educativa, ${ }^{14}$ Tiene la posibilidad de acceder a algún medio virtual para visualizar el informe del personal de UGEL sobre el monitoreo y acompañamiento que ha realizado en su Institución Educativa, ${ }^{15}$ El personal de UGEL utiliza medios virtuales para brindarle retroalimentación en relación al monitoreo y acompañamiento que ha realizado 
Carmen Rosa Sánchez-Tejada; Zila Isabel Esteves-Fajardo; Gladys Lola Luján-Johnson;

Mónica Mendoza-Gómez

en su institución educativa, ${ }^{16}$ Considera que la UGEL Piura debe implementar un sistema que permita sistematizar el monitoreo y acompañamiento a los directivos de las instituciones educativas.

\section{Análisis:}

El gráfico 3 Refleja las acciones de la innovación tecnológica de control y monitoreo para el monitoreo y acompañamiento en un orden de mayor a menor necesidad de atención por las respuestas mayoritarias de "nunca" y "a veces" por los directores de las instituciones educativas de la UGEL Piura designados y encargados: Primero Directivos y Docentes monitoreados y acompañados (I1: 100\%; I3: 100\%; 14: 100\%; 15:92\%)

\section{CONCLUSIÓN}

En relación a la gestión de las condiciones de la mejora de los aprendizajes, los directivos se han encontrado falencias en aspectos como: remisión de información a UGEL sobre los instrumentos de gestión y conformación de comisiones, uso de herramientas para la identificación de situaciones conflictivas, registro del monitoreo a los docentes sobre el cumplimiento de las horas efectivas de clase, uso de herramientas pertinentes y oportunas de procesamiento y organización de la información para la toma de decisiones en favor de la mejora de los aprendizajes y finalmente, en la implementación de mecanismos de transparencia y rendición de cuentas.

Se recomienda a los directivos de las instituciones educativas, trabajar en acuerdo con el personal docente, la implementación de herramientas de evaluación que permitan identificar fortalezas y debilidades de la labor pedagógica con el objetivo de optimizarla, proponiendo la ejecución proyectos de innovación pedagógica, orientados a la mejora de los aprendizajes. 
Carmen Rosa Sánchez-Tejada; Zila Isabel Esteves-Fajardo; Gladys Lola Luján-Johnson; Mónica Mendoza-Gómez

\section{REFERENCIAS CONSULTADAS}

Antúnez, S. (2000). La acción directiva en las instituciones escolares. Análisis y propuestas. Cuadernos de Educación. Barcelona: ICE-Horsori. 2a. ed. Barcelona: I.C.E Universitat Barcelona, 2000.Recuperado de: https://n9.cl/wzlg4

Bolívar, A. (2010). El liderazgo educativo y su papel en la mejora: una revisión actual de sus posibilidades y limitaciones. Psicoperspectivas: individuo y sociedad, 9(2), 933. Recuperdado de: https://n9.cl/ju7wi

Borden, A. (2015). Directores de Escuela en América Latina y el Caribe: ¿Líderes del Cambio o Sujetos a Cambio? Recuperado de: https://n9.cl/oezsa

Casassus, J. (2000). Problemas de la gestión educativa en América Latina (la tensión entre los paradigmas de tipo A y el tipo B). UNESCO.

Cuglievan, C. y Rojas, V. (2007). La gestión escolar en el marco de la autonomía: una mirada desde el cotidiano a cinco instituciones educativas estatales de Lima. GRADE. Recuperado de: https://n9.cl/cnoef

Guadalupe, C., León, J., Rodríguez, J., y Vargas, S. (2017). Estado de la educación en el Perú. Análisis y perspectivas de la educación básica. Impresiones y Ediciones Arteta E.I.R.L. Perú. Recuperado de: https://n9.cl/hlae

Dávila Newman, G. (2006). El razonamiento inductivo y deductivo dentro del proceso investigativo en ciencias experimentales y sociales. Laurus, 12(Ext),180-205. Recuperado de: https://n9.cl/nx847

Ministerio de Educación (2019) Resolución Ministerial Nº. 620-2019 MINEDU. Plan de gobierno digital del ministerio de educación para el periodo 2019 -2022. Lima Perú. Recuperado de: $\underline{\text { https://n9.cl/dl1eh }}$

Puiggrós, A. (2020). Balance del estado de la educación, en época de pandemia en América Latina. Editorial UNIPE Buenos Aires.

Schettini, P. y Pozner, P. (2001). Gestión educativa y escolar en el continente. Buenos Aires: OEA-Argentina.

Ugarte, M.; Arguedas, C., y Ángeles, N. (2012). Balance de la descentralización de la educación 2009-2012. Lima: Family Health International. 
Revista Interdisciplinaria de Humanidades, Educación, Ciencia y Tecnología

Año VII. Vol. VII. N³. Edición Especial III. 2021

Hecho el depósito de ley: pp201602FA4721

ISSN-L: 2542-3029; ISSN: 2610-802X

Universidad Nacional Experimental Francisco de Miranda (UNEFM). Santa Ana de Coro. Venezuela

Carmen Rosa Sánchez-Tejada; Zila Isabel Esteves-Fajardo; Gladys Lola Luján-Johnson;

Mónica Mendoza-Gómez

C2021 por los autores. Este artículo es de acceso abierto y distribuido según los términos y condiciones de la licencia Creative Commons Atribución-NoComercial-Compartirlgual 4.0 Internacional (CC BY-NC-SA 4.0)

(https://creativecommons.org/licenses/by-nc-sa/4.0/). 